\title{
PDK1 Activity Regulates Proliferation, Invasion and Growth of Hemangiomas
}

\author{
Ningning Zheng ${ }^{a} \quad$ Xudong Ding ${ }^{b}$ Amy Sun ${ }^{c}$ Rabita Jahanc \\ ${ }^{a}$ Department of Pathophysiology, China Medical University, ${ }^{b}$ Department of Anesthesiology, Shengjing \\ Hospital of China Medical University, International Education School, China Medical University, \\ Shenyang, China
}

\section{Key Words}

PDK1 $・$ Hemangioma $・$ Akt $・ \mathrm{mTOR} \cdot$ Vascular Tumor

\begin{abstract}
Background: Hemangiomas are common vascular endothelial cell tumors. Abnormally activated PI3K/Akt signaling pathway is one of the most important biological characteristics of Hemangioma. 3-phosphoinositide-dependent kinase 1(PDK1), an upstream protein of Akt, regulates the activity of Akt and its downstream kinases. The objective of this study is to explore the effect of PDK1 on malignant vascular tumors and their cell signaling mechanism in mice. Methods: Mouse Hemangioendothelioma Endothelial Cells (EOMA cells) and Nu/Nu mice were used. The silencing of PDK1 was mediated by lentiviral shRNA. Western blotting, WST-1 proliferation assay, Matrigel invasion assay, and Xenograft vascular tumor model were utilized to examine the effects and mechanism of PDK1 growth, proliferation, and invasion of an Hemangioma. Results: PDK1 deficiency significantly reduced the proliferation and invasion of EOMA cells in vitro, and depressed the growth of vascular tumor in vivo by decreasing the activity of Akt signaling pathway. Conclusion: We hypothesize that PDK1 plays a significant role in the progression and growth of vascular tumors and targeting PDK1 may thus be considered in their treatment.

Copyright (C) 2015 S. Karger AG, Basel
\end{abstract}

\section{Introduction}

Hemangiomas are common vascular endothelial cell tumors that appear with a wide range of clinical manifestation. It can start of as benign cases of infant Hemangiomas to low-

Ningning Zheng, PhD

KARGER 125
No.77 Puhe Road,Shenyang North New Area

Shenyang, Liaoning Province, 110122 (P.R. China)

Tel. +86 189-0091-0476, E-Mail nnzheng@mail.cmu.edu.cn 
graded malignant Hemangioendothelioma and Angiosarcoma, occurring more commonly in adults. In comparison to normal vascular endothelial cells, the proliferation rate and invasion capabilities of a vascular tumor endothelial cells are much greater.

Abnormal process of the signaling transduction pathway is an important biological characteristic of the tumor cells. For example, PI3K/Akt signaling pathway is crucial for maintaining the biological properties of the malignant cells. By activating PI3K/Akt signaling transduction pathway, the cell proliferation and differentiation are induced via a series of internal and external factors to avoid apoptosis [1-3]. Since this signaling pathway tends to be dysfunctional in most of the human tumors, it often becomes the target site for molecular cancer therapy.

As an upstream protein of Akt, 3-phosphoinositide-dependent kinase 1 (PDK1) regulates the activities of Akt and its downstream kinases [4]. Research has been found that PDK1mediated PI3K/Akt signaling pathway is associated with various types of cancers including melanoma, breast cancer, lung cancer, stomach cancer, prostate cancer, ovarian cancer, and pancreatic cancer [5]. PDK1 is over-expressed in most of the cancer cells [6]. The study has also showed Akt signaling pathway was over-activated in hemangiomas. Thus, inhibiting the expression of PDK1 followed by decreasing the activity of Akt may become a new treatment strategy for hemangioma.

Currently, there are only a few number of studies related to PDK1 in hemangioma. In this study, targeting PDK1 silencing via lentiviral-mediated shRNA demonstrated its effect and mechanism on the growth, proliferation and invasion of hemangioma, and thereby resulted a new method of therapy.

\section{Materials and Methods}

Antibodies and reagents

All the antibodies including Akt (\#9272), Phospho-Akt (Thr308) (C31E5E) (\#2965), PhosphoAkt (Ser473) (\#9271), PDK1 (\#3062), Phospho-S6 Ribosomal Protein (Ser235/236) (2F9) (\#4856) and $\beta$-Actin (8H10D10) (\#3700) were purchased from Cell Signaling Technology. VEGF (vascular endothelial growth factor) was purchased from R\&D System. Matrigel was purchased from BD Biosciences. WST-1 was purchased from Sigma.

\section{Cell culture}

Mouse Hemangioendothelioma Endothelial Cells (EOMA cells) were used and cultured in Dulbecco's modified Eagle's medium (DMEM) plus 4.5g/liter glucose supplemented with $10 \%$ fetal bovine serum (FBS) and antibiotics under $37^{\circ} \mathrm{C}$ and $5 \% \mathrm{CO}_{2}$. The culture medium was replaced once every two or three days. $293 \mathrm{~T}$ and about 80\%-confluence EOMA cells were washed with PBS and used in the experiments.

Lentiviral-mediated Short Hairpin RNA Silencing PDK1

Five short-hairpin RNA (shRNA) oligonucleotides were cloned into pLKO lentiviral expression system. The oligonucleotides encoding shRNA expression cassettes, targeting mouse PDK1, were as followed: \#H6(GCGGCTTTGTGATTTGTATTA),\#H7(GCCTGTTAGATTGGCAAATAT),\#H8(GCCGAAAGACATGACCACATT), \#H9(GCTGAGTATTTCTTTCAAGTT),\#H10(CGGCTTTGTGATTTGTATTAT). shPDK1, PAX2, VSVG, and a transfecting reagent, polyethylenimine (Polysciences) in DMEM media, were mixed into a $400-\mu l$ transfected container to generate lentivirus particles. The mixture was incubated for 15 minutes at room temperature, and then added to HEK 293 T cells ( $3 \times 10^{6}$ cells per 6 -cm plate), and then suspended in $3 \mathrm{ml} \mathrm{DMEM} / 10 \%$ FBS. $293 \mathrm{~T}$ cells were incubated in a viral incubator at $37^{\circ} \mathrm{C}$ for 48 hours. The supernatant containing lentivirus particles was collected and filtered through a $0.45-\mu \mathrm{m}$ syringe. For the viral transduction, hexadimethrine bromide (polybrene, $5 \mu \mathrm{g} / \mathrm{ml}$ ) (Sigma), added to the viral supernatant, was then transferred directly to EOMA cells $\left(1.5 \times 10^{6}\right.$ cells per 6 -cm plate). Four hours later, additional DMEM/10\%FBS was added to the cells, and the cells were cultured overnight. Transduced cells were subsequently selected from puromycin ( $2 \mathrm{ug} / \mathrm{ml}$ ) for 48 hours. pLKO-GFP was also transfected as the positive control.

\section{KARGER}




\section{Cellular Physiology Cell Physiol Biochem 2015;36:1903-1910 \begin{tabular}{l|l} 
and Biochemistry Published onIIne: July 17, 2015 & $\begin{array}{l}\text { C 2015 S. Karger AG, Basel } \\
\text { www.karger.com/cpb }\end{array}$ \\
\hline
\end{tabular} \\ Zheng et al.: Targeting PDK1 Inhibited Hemangioma}

Western Blot

Cells were washed in ice-cold phosphate-buffered saline, and whole-cell lysates were homogenized in the lysis buffer with $10 \mu \mathrm{L}$ Protease Inhibitor Cocktail (Sigma). Cell lysates were sonicated two times for 15 seconds and centrifuged at $14,000 \mathrm{~g}$ for 15 minutes at $4^{\circ} \mathrm{C}$. Clear lysates were then collected into a 1.5 -ml EP tube, and used to determine total protein levels. After diluting with sample buffer (Laemmli's SDS-Sample Buffer, Boston BioProducts), equal amounts of protein (typically $20 \mathrm{ug}$ ) were boiled for 3 minutes at $100^{\circ} \mathrm{C}$ and loaded onto 4-20\%Tris-Glycine gels (Invitrogen) with $20 \mathrm{ug}$ protein in each lane. After that, proteins were transferred to nitrocellulose membranes (BIO-RAD). Nonspecific reactions were blocked for 1 hour in $5 \%$ dry milk solution and Tris-buffered saline plus 0.1\% Tween-20 (TBST), and then incubated overnight with the appropriated primary antibody in $5 \%$ BSA/TBST under $4{ }^{\circ} \mathrm{C}$. Membranes were washed three times in TBST followed by incubation with the appropriated secondary horseradish peroxidase (HRP)-linked antibodies, and washed three times again. Membranes were developed with enhanced chemiluminescence reagents (Thermo Scientific, Rockford, IL), and exposed to film.

WST-1 cell proliferation assay

EOMA cells were divided into two groups: PDK1 silencing EOMA cells and pLKO control EOMA cells. According to the instructions of the manufacturer, $100 \mathrm{ul}$ per well of EOMA cells $\left(1 \times 10^{3} / \mathrm{ml}\right)$ were added in a 96-well plate, and incubated in complete medium for 48 hours. $10 \mu \mathrm{L}$ of WST-1 reagent was added to each well, and EOMA cells were incubated for another 4 hours. The absorbance of the samples was then measured at 490nm with a microplate reader (Molecular Device, California) after shaking for 1 min against the blank, a background control. Cell proliferation assays were in triplicates.

\section{Cell migration assay}

Transwell cell inserts with 8- $\mu \mathrm{m}$ pores were used. EOMA cells were also divided into two groups: PDK1 silencing EOMA cells and pLKO control EOMA cells. $100 \mu \mathrm{l}$ of Growth Factor-Reduced Matrigel (1mg/ $\mathrm{mL}$, BD Biosciences) was added into the upper chamber of transwell, and incubated for 4 hours at $37^{\circ} \mathrm{C}$ and $5 \% \mathrm{CO}_{2} .100 \mu \mathrm{L}$ of EOMA cells $\left(1 \times 10^{5} / \mathrm{ml}\right)$ was resuspended in DMED with $0.1 \% \mathrm{FBS}$, then seeded into the upper chamber. Lower chamber was filled with 600ul DMEM medium containing a chemoattractant (VEGF, $10 \mathrm{ng} / \mathrm{mL}$ ), and then cells were incubated for 24 hours. The unmigrated EOMA cells from the upper side of the inserts were scrapped, whereas the migrated EOMA cells were stained with Diff-Quick. After counting cells under the inverted microscope, the data was shown as the average of four high-power fields from triplicates after subtracting the average of cells migrating towards the medium without a chemoattractant.

Animals and Tumor Growth

Four- to six-week-old female $\mathrm{Nu} / \mathrm{Nu}$ mice were used in the experiments. Mice were divided into two groups: PDK1 silencing group and pLKO group. Vascular tumor model was introduced by injecting the cells subcutaneously in the flanks of mice $\left(2 \times 10^{5}\right.$ cells per site, two sites/mouse, 5 mice per group). Mice were under close observation daily to monitor their general conditions, and then euthanized after 2 weeks. The volume of the tumor was measured daily with a caliper.

\section{Statistical Analysis}

Results were expressed as the means \pm SE. The differences between the two groups were assessed by the two-tailed student's $t$ test. The differences between the means were considered as significant if $\mathrm{P}<0.05$.

\section{Results}

PDK1 over-expression in mouse hemangioma endothelial cells.

We examined the expression level of PDK1 presented in EOMA cells. High level of PDK1 was observed in EOMA cells compared with normal mouse lung and heart endothelial cells (Fig. 1). Furthermore, lower levels of PTEN (Phosphatase and tensin homolog, PTEN) were seen in EOMA cell [7]. These findings indicated common aberrations in the PI3K/PDK1/Akt pathway, which may have contributed to the formation of hemangioma. 


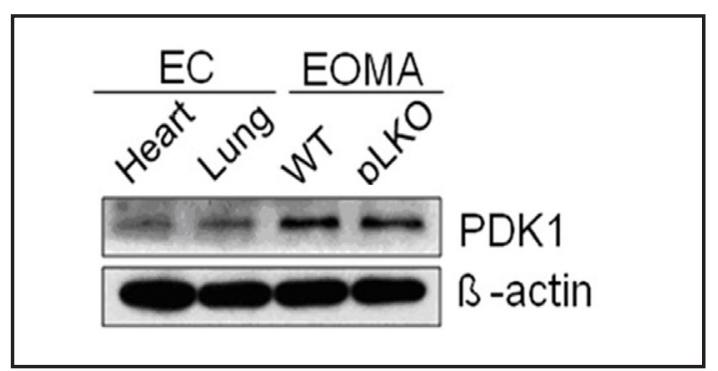

Fig. 1. Western blot analysis of normal mouse ECs and EOMA cells for PDK1. Increased PDK1 expression occurred in EOMA cells with $\beta$-actin as protein loading control.

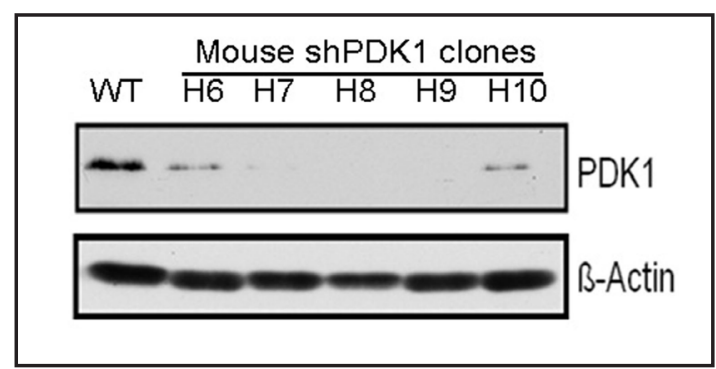

Fig. 2. PDK1 silencing in EOMA cells by lentiviral short-hairpin RNA. Five separate shPDK1 clones \#H6,\#H7,\#H8,\#H9 and \#H10 were tested. shPDK1 \#H8 was selected.

Fig. 3. Monolayer EOMA cells of lentiviral shRNA transfection by GFP, pLKO and PDK1 under optical microscope.

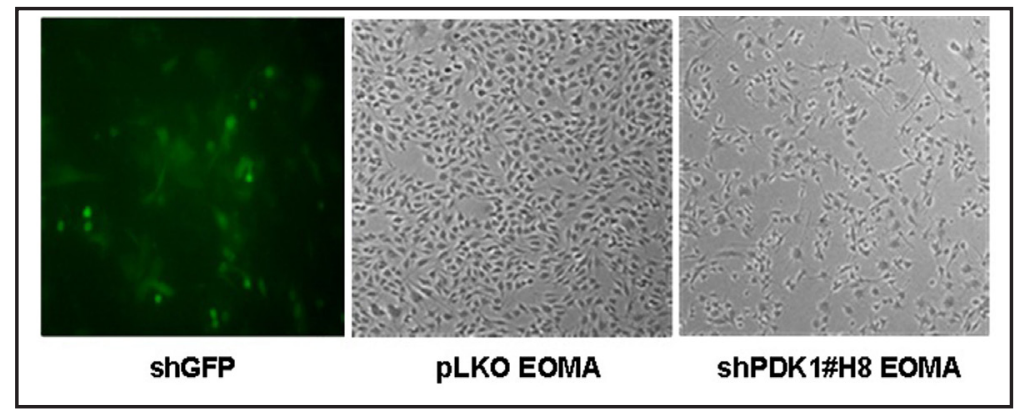

The specificity of lentiviral-mediated shRNA inhibits the expression of PDK1

By using GFP as the positive control, it was proven that lentiviral gene silencing expression vector was constructed successfully with the stable ability of RNA interference. Western blot results suggested that shPDK1 clones inhibited the expression levels of the target proteins (Fig. 2 and 3). In this experiment, EOMA cells transfected by shPDK1\#H8 were used as the cell strain to stabilize PDK1 silencing expression.

Deficiency of PDK1 significantly reduced the proliferation of EOMA cells

WST-1 colorimetric experiment was performed with stabilized PDK1 silencing EOMA cells to examine the effect of PDK1 on the proliferation of hemangioma cells. The cells were divided into two groups: PDK1 silencing EOMA cells and pLKO EOMA cells. $1 \times 10^{3}$ cells/well were inoculated in a 96-well culture plate with the volume of $100 \mu \mathrm{l} /$ well. Each group contains triplicates and one blank left as the control (adding medium only). After incubating for 48 hours, WST-1 was added. The samples were then incubated for another 4 hours. After zeroing with the blank, the absorbance (OD value) of each well was measured on a microplate reader at $490 \mathrm{~nm}$, and the relative OD ratio was used to indicate the cell proliferation capacity. The average was taken among the three-replicate wells in each group. By comparing with pLKO group, the proliferation capacity of EOMA cells in shPDK1 group was significantly reduced (Fig. 4, $\mathrm{P}<0.05$ ).

Deficiency of PDK1 significantly reduced the migration of EOMA cells

In order to detect how migration ability of EOMA cells changed after silencing of PDK1, EOMA-cell concentration was adjusted to $5 \times 10^{5}$ cells $/ \mathrm{ml}$ for both pLKO and shPDK1 groups, and their migration abilities were tested through transwell assay. After close observation under a microscope, three high-power fields were randomly selected, and the numbers of cells that passed through Matrigel were counted. By comparing with pLKO control group, the result showed that the numbers of migrated cells in shPDK1 group were significantly reduced (Fig. 5, P <0.05). It further explained that the inhibition of PDK1 expression could significantly reduce the migration ability of hemangioma cells. 


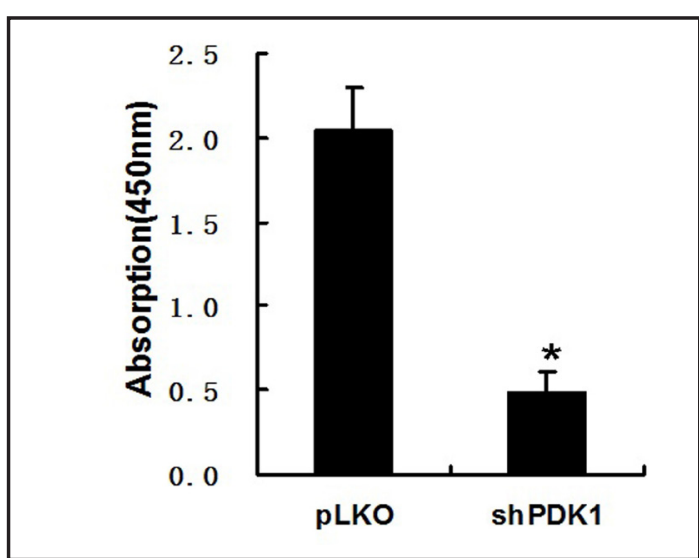

Fig. 4. EOMA cells with PDK1 silencing affected cell proliferation. shPDK1 inhibited EOMA cell proliferation compared with pLKO control $(\mathrm{p}<0.05)$.

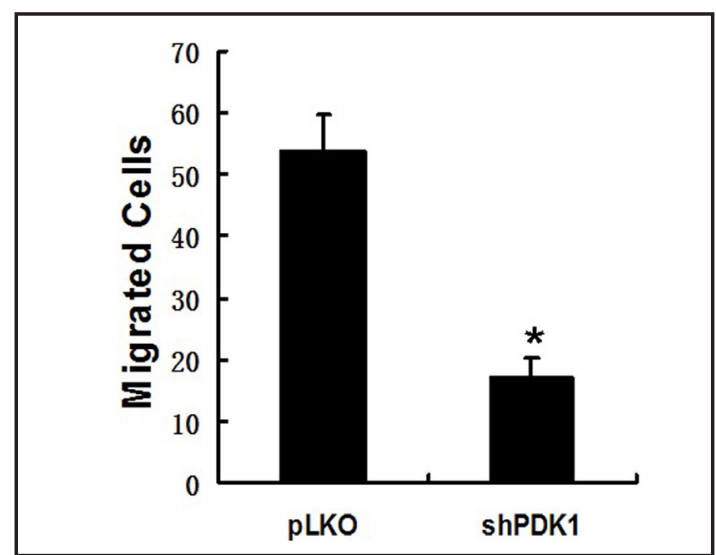

Fig. 5. EOMA cells with PDK1 silencing affected cell migration. shPDK1 inhibited EOMA cell migration compared with pLKO control $(\mathrm{p}<0.05)$.

Fig. 6. Western blot analysis of knocking down PDK1 in EOMA cells for AKT signaling pathway. Decreased phosphorylated AKT473, AKT308 and S6 occurred with $\beta$-actin as protein loading control $(\mathrm{p}<0.05)$. Wortmannin $(50 \mathrm{nM})$ was treated as positive control.

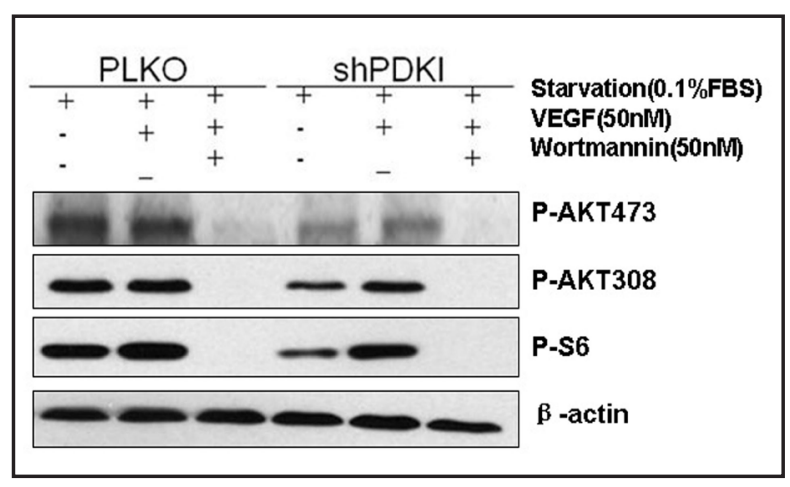

Silencing of PDK1 inhibited the activity of Akt signaling pathway in EOMA cells

In order to investigate how PDK1 regulated the growth, proliferation, and invasion of hemangioma, the activation of Akt signaling pathway was examined. The cells were divided into two groups: pLKO and PDK1 silencing groups. The cells in each group were treated with three different ways: cell starvation treatment (cells were cultured in DMEM medium containing 0.1\% FBS and incubated for 12 hours), VEGF treatment (cultured in 50nM VEGF and incubated for 10 minutes), and wortmannin treatment (cultured in $50 \mathrm{nM}$ wortmannin and incubated for 12 hours). Wortmannin is an inhibitor of PI3K, which was chosen as the

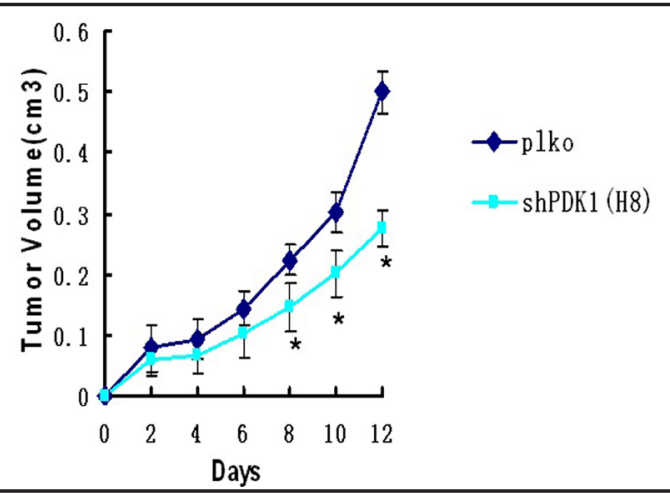

Fig. 7. EOMA cells with PDK1 silencing injected subcutaneously affected tumor growth in nu/nu mice. Tumor size was monitored for 12 days. PDK1 silencing significantly reduced tumor growth as compared to pLKO control from day8 to day12 (p<0.05). positive control. After all the treatments, cell lysis solution was collected and the protein concentration was measured using Western Blot. After comparing with the control group, the result showed that the phosphorylation levels of Akt (Ser473), Akt (Thr308), and S6 in PDK1 silencing EOMA cells were all declined. Silencing PDK1 did not only decrease the phosphorylation of Akt (Thr308), but also reduced the phosphorylation levels of Ser473 and S6. Therefore, reduction of PDK1 gene expression could result in the decrease of Akt 


\section{Cellular Physiology Cell Physiol Biochem 2015;36:1903-1910

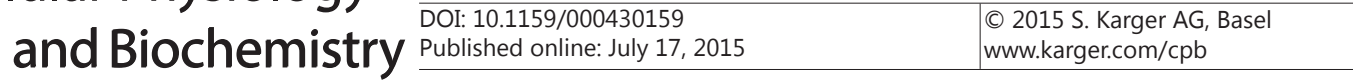 \\ Zheng et al.: Targeting PDK1 Inhibited Hemangioma}

signaling pathway activity followed by the inhibition of the downstream target protein function hindering the growth of hemangioma cells (Fig. 6).

Silencing of PDK1 inhibited the growth of hemangioma in mice

The outcome of inhibiting PDK1 expression on hemangioma growth on mice was examined by establishing a subcutaneous hemangioma transplantation model. EOMA cells of pLKO and PDK1 silencing groups were injected subcutaneously into the flank of the nude mice respectively. The general conditions of the mice were closely observed, the tumor size was measured the following day. The volume of the tumor was calculated to be $0.53 x$ lengthxwidth ${ }^{2}$. In comparison to the pLKO control group, the result showed that the volume of the tumor formed by PDK1 silencing group had significantly reduced (Fig. 7, $\mathrm{P}<0.05)$. The inhibition of PDK1 expression was able to significantly reduce the growth rate of hemangioma, and possibly prevent the formation of them on nude mice.

\section{Discussion}

The study has found that the abnormal activation of PI3K/Akt signaling pathway is a parallel consequence of the development of hemangioma. Therefore, this pathway could be an excellent target site for newly developed molecular hemangioma therapies. PI3K/Akt signaling pathway act on a series of cellular processes and reactions including gene expression regulation, cell cycle regulation, cell proliferation, differentiation, and so on [8-10]. Akt is a key protein kinase of this pathway, which regulates multiple downstream pathways such as MMP-2 (matrix metalloproteinase-2), IKK (inhibitor of nuclear factor kappa-B kinase), P21, and mTOR. As a result, cell proliferation, growth, anti-apoptosis and migration all take place. PDK1 is a kind of serine / threonine protein kinase. Research has showed that IGF1 (Insulinlike Growth Factor 1) can activate Akt in the normal cells, but cannot do so in PDK1-deficient cells [11]. PDK1 is an important upstream Akt activation protein kinase in mammalian cells, and can regulate series of the biological reactions by PI3K/Akt signaling pathway [12, 13]. As a central hub for many crucial cellular signaling pathways, PDK1 is widely found in many tissues, and plays a vital role in the tumor cell growth, anti-apoptosis, angiogenesis $[14,15]$. Our study found that stable PDK1 silencing hemangioma vascular endothelial cells slowed down the growth, decreased proliferation and invasion abilities in vitro. The experiment of nude mice in vivo further confirmed that PDK1 silencing could significantly inhibit the growth of hemangiomas. These results presented that PDK1 was closely related to the growth, proliferation, and invasion of hemangioma. The study also illustrated that the silencing of PDK1 was associated with the inhibition of the phosphorylation in Akt and its downstream kinase S6. PDK1 is the upstream kinase of Akt at Thr308, therefore, it was evident that down-regulating PDK1 lead to the decrease of the phosphorylation levels of Akt at Thr308. However, in our study we also found a decrease in the level of phosphorylation of Akt at Ser473. Studies have said that the kinase responsible for phosphorylating Ser473 has been elusive for a long time and a range of different candidates has been proposed including Akt itself [16], MAPKAP kinase 2 (mitogenactivated protein kinase-activated protein kinase 2) [17], modified PDK1 [18], ILK (integrin-linked kinase) [19] and conventional PKCs (protein kinase C isoforms) [20]. Those studies also demonstrated that phosphorylation of Akt Thr308 and Akt Ser473 levels had substantially been reduced in PDK1-deficient platelets [21]. The same result was proven in our study that PDK1-deficient induced the decrease of the phosphorylation Akt at Ser473 in EOMA cells and thereby participated in the inhibition of the tumor cell growth. In conclusion, PDK1 may become a new target of hemangioma treatment. However, further studies are required to understand the detailed mechanism of the occurrence, development, and metastasis of hemangioma. 


\section{Cellular Physiology Cell Physiol Biochem 2015;36:1903-1910 \begin{tabular}{ll|l} 
and 10.1159/000430159 & $\begin{array}{l}\text { O 2015 S. Karger AG, Basel } \\
\text { www.karger.com/cpb }\end{array}$ \\
\hline
\end{tabular}}

Zheng et al.: Targeting PDK1 Inhibited Hemangioma

\section{Disclosure Statement}

The authors declare no conflict of interest.

\section{Acknowledgments}

We would like to thank Laura E Benjamin and Qi Xue for help with advice and experimental materials.

\section{References}

1 Haga S, Ozaki M, Inoue H, Okamoto Y, Ogawa W, Takeda K, Akira S, Todo S: The survival pathways phosphatidylinositol-3 kinase (PI3-K)/phosphoinositide-dependent protein kinase 1 (PDK1)/Akt modulate liver regeneration through hepatocyte size rather than proliferation. Hepatology 2009;49:204214.

-2 Patsoukis N, Brown J, Petkova V, Liu F, Li L, Boussiotis VA:Selective effects of PDK1 on Akt and Ras pathways regulate molecular components of the cell cycle and inhibit T cell proliferation. Sci Signal DOI: $10.1126 / 2002796$.

- 3 Limon JJ, Fruman DA: Akt and mTOR in B Cell Activation and Differentiation. Front Immunol 2012;3:228.

4 Matheny RW, Adamo ML: Current perspectives on Akt Akt-ivation and Akt-ions. Exp Biol Med (Maywood) 2009;234:1264-1270.

5 Raimondi C, Falasca M: Targeting PDK1 in cancer. Curr Med Chem 2011;18:2763-2769.

-6 AbdulHameed MD, Hamza A, Liu J, Zhan CG: Combined 3D-QSAR modeling and molecular docking study on indolinone derivatives as inhibitors of 3-phosphoinositide-dependent protein kinase-1. J Chem Inf Model 2008;48:1760-1772.

7 Zheng NN, Ding XD, Zhang HP: Targeting rictor inhibits mouse vascular tumor cell proliferation and invasion in vitro and tumor growth in vivo. Neoplasma 2013;60:41-45.

8 Tsuchiya A, Kanno T, Nagaya H, Shimizu T, Tanaka A, Nishizaki T: PTP1B inhibition causes Rac1 activation by enhancing receptor tyrosine kinase signaling. Cell Physiol Biochem 2014;33:1097-1105.

-9 Shibata E, Kanno T, Tsuchiya A, Kuribayashi K, Tabata C, Nakano T, Nishizaki T: Free fatty acids inhibit protein tyrosine phosphatase 1B and activate Akt. Cell Physiol Biochem 2013;32:871-879.

10 Niba ET, Nagaya H, Kanno T, Tsuchiya A, Gotoh A, Tabata C, Kuribayashi K, Nakano T, Nishizaki T: Crosstalk between PI3 kinase/PDK1/Akt/Rac1 and Ras/Raf/MEK/ERK pathways downstream PDGF receptor. Cell Physiol Biochem 2013;31:905-913.

11 Liu H, Song G, Zhou L, Hu X, Liu M, Nie J, Lu S, Wu X, Cao Y, Tao L, Chen L, Qian L: Compared analysis of LncRNA expression profiling in pdk1 gene knockout mice at two time points. Cell Physiol Biochem 2013;32:1497-1508.

12 Bayascas JR: PDK1: the major transducer of PI 3-kinase actions. Curr Top Microbiol Immunol 2010;346:929.

13 Vanhaesebroeck B, Alessi DR: The PI3K-PDK1 connection: more than just a road to PKB. Biochem J 2000;346:561-576.

14 Westmoreland JJ, Wang Q, Bouzaffour M, Baker SJ, Sosa-Pineda B: Pdk1 activity controls proliferation, survival, and growth of developing pancreatic cells. Dev Biol 2009;334:285-298.

15 Shiojima I, Walsh K: Role of AKT signaling in vascular homeostasis and angiogenesis. Circ Res 2002;90:1243-1250.

16 Toker A, Newton AC: Akt/protein kinase B is regulated by autophosphorylation at the 2hypothetical PDK-2 site. J Biol Chem 2000;275:8271-8274.

17 Alessi DR, Andjelkovic M, Caudwell B, Cron P, Morrice N, Cohen P, Hemmings BA: Mechanism of activation of protein kinase B by insulin and IGF-1. EMBO J 1996;15:6541-6551.

18 Leslie NR, Biondi RM, Alessi DR: Phosphoinositide-regulated kinases and phosphoinositide phosphatases. Chem Rev 2001;101:2365-2380. 


\section{Cellular Physiology Cell Physiol Biochem 2015;36:1903-1910}

and Biochemistry $\frac{\text { DOI: 10.1159/000430159 }}{\text { Publisned onIIne: July 17, } 2015} \underset{\text { Zheng et al.: Targeting PDK1 Inhibited Hemangioma }}{ }$

-19 Balendran A, Casamayor A, Deak M, Paterson A, Gaffney P, Currie R, Downes CP, Alessi DR: PDK1 acquires PDK2 activity in the presence of a synthetic peptide derived from the carboxyl terminus of PRK2. Curr Biol 1999;9:393-404.

20 Kroner C, Eybrechts K, Akkerman JW: Dual regulation of platelet protein kinase B. J Biol Chem 2000;275:27790-27798.

21 Chen X, Zhang Y, Wang Y, Li D, Zhang L, Wang K, Luo X, Yang Z, Wu Y, Liu J: PDK1 regulates platelet activation and arterial thrombosis. Blood 2013;121:3718-3 